\section{Is measurement of blood pressure worthwhile in the diabetic eye clinic?}

\begin{abstract}
Purpose The UK Prospective Diabetic Study has confirmed the importance of blood pressure (BP) as a major risk factor for diabetic retinopathy (DR). We wanted to investigate whether measuring the BP in the diabetic eye clinic could identify new hypertensive patients and monitor control in existing ones. Patients and methods We compared BP in patients attending the diabetic eye clinic with home blood pressure measurement (HBPM) and ambulatory BP measurement (ABPM). In all, 106 patients attending a diabetic eye clinic were selected at random from clinic attendees. BP measurement (on an Omron 705 CP) was performed in the eye clinic and also compared to HBPM three times per day with an Omron 705 CP machine, and was compared to diabetic clinic measurements. In addition, 11 randomly chosen patients had $24 \mathrm{~h}$ ABPM to validate the
\end{abstract} above techniques.

Results In all, 106 patients (70 male and 36 female) were recruited for the study, of which 71 were known to be hypertensive on antihypertensive medication. Of the total, 75 patients $(70.8 \%)$ had $\mathrm{BP}>\mathbf{1 4 0 / 8 5}$ in the eye

Departments of Ophthalmology and Diabetes, Birmingham Heartlands and Solihull NHS TRUST Birmingham, UK

Correspondence: PM Dodson

Undergraduate Centre Birmingham Heartlands Hospital Bordesley Green East Birmingham B9 5SS, UK Tel: + 4401214242000

Fax: + 4401214240593

E-mail:paul.dodson@

heartsol.wmids.nhs.uk

Received: 11 May 2003 Accepted: 15 January 2004 Published online: 23 July 2004 clinic, of which $51(68 \%)$ were known to be hypertensive on treatment and this was confirmed in $46(90 \%)$ on HBPM. A total of, 24 patients $(22.6 \%)$ were newly diagnosed as hypertensive in the eye clinic, which was confirmed by HBPM in 22 patients $(92 \%)$. The mean BP of the measurements performed in the eye clinic was significantly higher than that carried out in the diabetic clinic $(P<0.01)$. Tropicamide $1 \%$ and phenylephrine $2.5 \%$ eye drop instillation had no effect on BP. In 11 randomly chosen patients, $24 \mathrm{~h}$ ABPM validated both diabetic eye clinic and home BP measurements.

Conclusion Attendance at the diabetic eye clinic is an important chance to detect both new patients with systemic hypertension and those with inadequate BP control.

\section{S Al-Husainy, J Farmer, JM Gibson and PM Dodson}

Ophthalmologists should be encouraged to measure BP in their diabetic patients attending diabetic eye clinics, as it is an important risk factor for DR. On the basis of our findings, good BP control is a goal yet to be achieved in diabetic patients with retinopathy. Eye (2005) 19, 312-316. doi:10.1038/sj.eye.6701480 Published online 23 July 2004

Keywords: diabetic retinopathy; hypertension; Diabetic eye clinic blood pressure measurement; home blood pressure measurement; $24 \mathrm{~h}$ ambulatory blood pressure measurement; mydriasis

Introduction

Hypertension is a major risk factor in the development and progression of microvascular complications of diabetes. ${ }^{1}$ It is present in $70 \%$ of patients with type II diabetes. ${ }^{2}$

The United Kingdom Prospective Diabetes Study (UKPDS) has confirmed the crucial role of blood pressure (BP) management in diabetic subjects. ${ }^{3}$ There was a $37 \%$ reduction in the risk of microvascular end-point damage,

predominantly owing to a reduced risk of retinal photocoagulation, and a $47 \%$ reduction in the risk of deterioration of visual acuity by three lines, in those patients assigned to tight BP control $(144 / 82 \mathrm{mmHg})$ compared with the group assigned to less tight BP control (154/ $87 \mathrm{mmHg}$ ). In all, $29 \%$ of patients in the tight BP control group required three or more antihypertensive medications. ${ }^{3}$ These data, in addition to those from other landmark studies, have led to recommendations of BP targets, particularly systolic pressure of $<140 \mathrm{mmHg}$ in diabetic subjects. ${ }^{4}$

Patients with diabetic retinopathy (DR) attend ophthalmology services for ophthalmic care, giving an opportunity for BP measurement and discussion of the importance of control of risk factors for DR. BP measurement in this clinic 
setting is usually performed by the nursing staff on new patients and subsequently opportunistically. Is this worthwhile, considering the many variables affecting BP measurement, including differing equipment, the Hawthorne (BP reduction with repeated measurement) and white coat effects?

We have therefore performed a study to determine if the measurement of BP in the diabetic eye clinic setting does provide a useful clinical record of BP status, and therefore whether this may or may not influence subsequent management. A secondary aim was to review cardiovascular therapies and achievement of cardiovascular treatment targets in our DR cohort.

\section{Patients and methods}

Patients were recruited randomly from the attendees at the diabetic eye clinic of Birmingham Heartlands Hospital, and consent was obtained for the study protocol. Measurement of BP was performed by the electronic Omron 705 CP machine, which has been validated by the British Hypertension Society (BHS). ${ }^{5}$ The mean of two readings, after $5 \mathrm{~min}$ rest in the sitting position, with a $20 \mathrm{~min}$ interval, was taken as the BP clinic reading at attendance and repeated again 1 week later, when the Omron $705 \mathrm{CP}$ machines allocated to patients for home monitoring were returned. All initial and subsequent diabetic eye clinic BP measurements were performed by one operator (SAH).

On the recruitment visit, patients were further randomly allocated into two groups: the first had BP measured prior to the instillation of mydriatic eye drops (one drop of both Tropicamide 1\% and 2.5\% phenylephrine), and in the second group, BP recording was made $30 \mathrm{~min}$ after eye drop instillation.

All patients were provided with Omron devices at home, and instructed to measure their own BP at home (home blood pressure monitoring, HBPM) three times/ day (morning, afternoon, and evening) for one week, ${ }^{6}$ allowing at least $10-15 \mathrm{~min}$ rest prior to each measurement, and asked to return the device the subsequent week with recordings to the eye clinic. At this attendance, a further clinic measurement of BP was performed. In order to validate the BP measurements (particularly the HBPM), 10\% of the patients were selected at random to have $24 \mathrm{~h}$ ambulatory blood pressure monitoring (ABPM), using a Space Labs Device 90121, which has been validated by the BHS. ${ }^{7}$

Patients excluded from the study were those with diabetic nephropathy, and with arm circumference $>33 \mathrm{~cm}$, who would have necessitated a different cuff size. BP measurement at the last attendance for diabetic medical review (most commonly from the hospital diabetic clinic annual review measured by an Omron 705
CP machine) was recorded from their hospital records, to allow comparison to the diabetic eye clinic study recordings.

The patient's treatment regimen for diabetes, hypertension, cholesterol lowering, and use of aspirin was recorded, as well as the type and treatment of DR (eg laser treatment), and the most recent glycated haemoglobin and cholesterol levels.

Approval was obtained from the local ethics committee and the project was registered with the research database of Birmingham Heartlands Hospital.

Statistical analysis was performed using paired and unpaired $t$-tests, and $\chi^{2}$-test for distribution.

\section{Definitions}

Hypertension in patients with diabetes mellitus was defined according to the guidelines of the World Health Organisation/International Society of Hypertension and the BHS as $>140 / 90 \mathrm{mmHg}$ in the clinic, and inadequate control of BP (audit standard) as $>140 / 85 \mathrm{mmHg} .{ }^{4}$ The guidelines suggested that home and ambulatory BP should be $<130 / 75 \mathrm{mmHg}$ (or $12 / 7 \mathrm{mmHg}$ less than the clinic recording). ${ }^{4}$ Other targets for cardiovascular risk factor management were glycated haemoglobin $<7 \%$, and a random serum cholesterol level of $<5.2 \mathrm{mmol} / 1$. In view of the recommendations that all patients with DR aged $>30$ years should be on aspirin therapy, ${ }^{8}$ and the high cardiovascular risk of diabetic patients commonly requiring statin therapy, ${ }^{9}$ these data were recorded but only referred to current treatment, not previous or subsequent.

\section{Results}

A total of 106 diabetic patients with retinopathy were recruited, and the clinical details are shown in Table 1. In all, 89 (84\%) patients had type II diabetes, and the most common retinopathy was maculopathy $(65 \%)$. All patients had sight threatening DR (maculopathy, preproliferative and proliferative forms), with $74 \%$ having had laser therapy prior to the study. $21(19.8 \%)$ of patients were attending primary care only, with attendance at the diabetic eye clinic their first in secondary care.

\section{$B P$ recordings}

$\mathrm{BP}$ was recorded in 58 patients prior to the instillation of mydriatic drops, and in 48 patients, it was measured $30 \mathrm{~min}$ after. There was no significant difference in BP recordings in these two groups (differences in mean values: systolic 4.2 and diastolic $0.85 \mathrm{mmHg}$ ) as shown in Table 2 . 
Table 1 Clinical details of the patients with DR studied $(n=106)$

\begin{tabular}{lc}
\hline Parameter recorded & Details \\
\hline Mean age (years) & 61.61 (range: $28-87)$ \\
Diabetes mellitus: type I/II & $17 / 89$ \\
Mean duration of diabetes (years) & 17.2 \\
Patients known to be hypertensive on medication $(n)^{\mathrm{a}}+$ & 71 \\
Patients on no treatment (presumed normotensive) $(n)^{\mathrm{a}}+$ & 35 \\
Insulin/oral hypoglycaemics & $65 / 38$ \\
Diabetic care: hospital/primary care only & $85 / 21$ \\
Type of DR $(n)^{\mathrm{b}}$ & $14(14.84 \%)$ \\
Preproliferative & $26(27.56 \%)$ \\
Proliferative & $61(64.66 \%)$ \\
Maculopathy & $70(74.20 \%)$ \\
Patients treated with laser & \\
\hline
\end{tabular}

${ }^{\mathrm{a}}$ From records of last attendance to diabetic clinics prior to study period.

${ }^{b}$ Recorded from the diabetic eye clinic records (JMG and PMD).

Table 2 Results of BP measurements

\begin{tabular}{lccc}
\hline Type of measurement & Patient number & Systolic BP $(\mathrm{mmHg})$ & Diastolic BP $(\mathrm{mmHg})$ \\
\hline Eye clinic visit (initial) & 106 & ${ }^{\mathrm{a}} 156.6 \pm 21.7$ & $86.80 \pm 11.9$ \\
With mydriatics & 48 & 154.22 & 86.33 \\
Without mydriatics & 58 & 158.48 & 87.18 \\
Eye clinic visit 2 (1 week after initial visit) & 77 & 155.4 & 76.7 \\
Home BP measurement & 106 & 147.7 & 81.8 \\
Difference in means to eye clinic BP value. & 106 & -8.8 & -4.97 \\
(- mean mmHg) & 104 & ${ }^{\mathrm{a}} 147.8 \pm 22.5$ & $79.5 \pm 11.6$ \\
Last BP of routine diabetic clinic & &
\end{tabular}

Figures $=$ means \pm SD.

${ }^{a} P<0.01$ by paired $t$-test, comparing diabetic eye clinic BP compared to last BP record in the diabetic clinic.

Table 3 Comparison of BP status by diabetic eye clinic and HBPM

\begin{tabular}{lcccc}
\hline Categoray & $\begin{array}{c}\text { Mean BP } \\
(\text { mmHg) }\end{array}$ & Number of patients with BP above BHS guidelines (4) & $\begin{array}{c}\text { Number normotensive by } \\
\text { BHS guidelines (4) }\end{array}$ \\
\cline { 3 - 5 } & & Eye clinic & HBPM & 20 \\
\hline Known hypertensives $n=71$ & $160 / 87$ & $51 / 71(71.8 \%)$ & $46 / 71(64 \%)$ & 11 \\
Presumed normotensives $n=35$ & $150 / 84$ & $24 / 35(68.6 \%)$ & $22 / 35(62.8 \%)$ & 20 \\
\hline
\end{tabular}

The mean BP of all patients attending the diabetic eye clinic was $156.5 / 86.8 \mathrm{mmHg}$, which was significantly higher than that recorded at the diabetic clinic outpatient attendance prior to the study $(147.7 / 79.5 \mathrm{mmHg}: P<0.01$ for systolic pressure difference). Of the $77 / 106$ patients who attended for BP measurement a week later in the diabetic eye clinic, there was no significant difference in mean BP values $(155.4 / 76.7 \mathrm{mmHg})$ compared to initial values taken 1 week earlier.

The HBPM performed by all patients demonstrated mean BP values of $147.7 / 81.8 \mathrm{mmHg}(8.8 / 5 \mathrm{mmHg}$ less than initial recordings in the diabetic eye clinic).

\section{Hypertension and therapies}

In all, 71 patients $(67 \%)$ were known to be hypertensive, on antihypertensive medication, prior to the study in the diabetic eye clinic. A total of $12(17 \%)$ were on three, 26 (36\%) on two, and $33(46 \%)$ on one antihypertensive agent. In all, $34 \%$ were on an ACE inhibitor, and 23\% were on calcium channel blocking agents. The mean BP recordings of these patients were $160 / 87 \mathrm{mmHg}$. However, $51(72 \%)$ of these patients had diabetic eye clinic BP recordings above the target or audit standard of $<140 / 85 \mathrm{mmHg}$. These findings of BP values above target were confirmed in 46 of $51(90 \%)$ by HBPM (shown in Table 3).

The remaining 35 patients (33\% of the total 106 patients) had previously been recorded as normotensive (therefore not requiring antihypertensive medication) at the diabetic clinic attendance prior to the study. The mean BP of these patients was $150 / 84 \mathrm{mmHg}$, reflecting 24 cases of previously unidentified hypertension $(68.6 \%)$, with these findings confirmed by HBPM in 22 of the 24 
Table 4 Comparison of BP status by HBPM and 24h ABPM

\begin{tabular}{lccccc}
\hline Category & Patient number & $\begin{array}{c}\text { Mean BP daytime } \\
\text { ABPM (mmHg) }\end{array}$ & $\begin{array}{c}\text { Mean HBPM } \\
(\text { mmHg) }\end{array}$ & $\begin{array}{c}\text { Within BHS } \\
\text { guidelines }\end{array}$ & $\begin{array}{c}\text { Agreement of HBPM } \\
\text { with ABPM }\end{array}$ \\
\hline Known hypertensives & 8 & $140 / 75$ & $151 / 83$ & 4 & $8(100 \%)$ \\
Presumed normotensive & 3 & $146 / 84$ & $150 / 89$ & 1 & $3(100 \%)$ \\
\hline
\end{tabular}

No significant differences between the mean BP values and 100\% concordance between HBPM and ABPM.

patients (92\%) (shown in Table 3). Only 11 of these previously presumed normotensive patients in diabetic clinics (31\%) were indeed normotensive in this study in the diabetic eye clinic.

A $24 \mathrm{~h}$ ABPM was performed in 11 patients, of whom eight were known hypertensive patients on medication. These BP recordings, shown in Table 4, confirm the results of HBPM, demonstrating complete agreement in the differentiation of patients with BP levels above and below target levels, as well as those with a new diagnosis of hypertension. Therefore, ABPM confirmed and validated the BP results of HBPM in all 11 patients.

\section{Other targets and therapies}

Drug therapy was recorded in all 106 diabetic patients studied. Current therapies included aspirin in only $36 \%$, and statin treatment in 35\%. Only $38 \%$ of patients had a glycated haemoglobin level below the target of $<7 \%$, but $62 \%$ had a random serum cholesterol level $<5.2 \mathrm{mmol} / \mathrm{l}$, and $>8.5 \mathrm{mmol} / 1$ in $8.5 \%$.

\section{Discussion}

The relationship between increasing BP and the development and progression of diabetic retinopathy (DR) is now well established, with retinal hyperperfusion and shunting a direct cause of damage to retinal capillaries. ${ }^{1,3,10}$ Intensive intervention with tight control of $\mathrm{BP}<140 / 80$ in patients with type II diabetes has been consistently shown to reduce progression of retinopathy and laser requirement in large randomised controlled trials, such that the benefit from BP lowering was even greater than that of intensive glycaemic control.,11 The evidence base for benefit of antihypertensive treatment for DR in type I diabetes is not as well studied, but is suggested in one large randomised controlled study (EUCLID) of ACE inhibition. ${ }^{12}$ The results of the recent large hypertension trials have led to national guidelines, defining targets and audit standards for optimal BP control, as well as lowering the threshold of BP formerly used in the definition of hypertension (BHS, 1999). 4,13
With such importance placed on the treatment of hypertension in the management of DR, several questions are raised:

(1) Should BP measurements be made in medical ophthalmology/diabetic eye clinics?

(2) Is it clinically worthwhile to perform?

(3) Would BP measurement, in this setting, add information that is not already available in diabetic clinics? Of relevance to the latter is that approximately $50 \%$ of diabetic patients have diabetes management in primary care and attendance at the hospital diabetic eye clinic may be the first presentation to secondary care services.

Our study has demonstrated that mean BP measured in patients attending the diabetic eye clinic was significantly higher than that of routine diabetic clinic measurements, and this was of the order that may be clinically important $(+9 \mathrm{mmHg}$ in systolic pressure), including the potential to alter therapeutic management. Our observations suggest that this difference was not due to topical mydriasis. Other explanations include the 'white coat effect' or anxieties relating to practice in diabetic eye clinics. Possible factors include clinic logistics (eg environment, waiting times), concerns about laser treatment, visual failure, and blindness, the most feared complication of diabetes. However, the data suggest negligible Hawthorne effect (BP reduction with repeated measurement), as mean BP levels at entry were not significantly changed at the repeat measure 1 week later. In contrast, the HBPM results, confirmed by $24 \mathrm{~h}$ $A B P M$ values in a random patient sample, suggest that the diabetic eye clinic BP levels may have been a more accurate record of actual $\mathrm{BP}$, rather than the casual record in diabetic clinics in primary and secondary care.

A measure of BP in the diabetic eye clinic therefore appears to be clinically useful, identifying many patients with inadequate BP control, and identifying one-third of normotensive patients (ie not previously on antihypertensive therapy) as hypertensive and appropriate therapy subsequently commenced. Compared to UKPDS and HOT study drug treatment 
requirements to achieve target $\mathrm{BP}$, it is clear that a large number of patients are not receiving sufficient combinations of two or three antihypertensive agents to achieve tight BP control $(<140 / 85 \mathrm{mmHg})^{3,13,14}$

With modern emphasis on evidence-based targets for other aspects of diabetes management, our data demonstrate that the majority of subjects with type II diabetic attending our diabetic eye clinic had not achieved a glycated haemoglobin target of $<7 \%$, and only a third of patients were on aspirin therapy; the latter has been recommended as appropriate for all patients with DR $>30$ years of age. ${ }^{8}$ One-third of patients in our cohort were on statins. This again demonstrates likely undertreatment in the cohort, as statin treatment is now indicated for diabetic patients with a 10 year coronary risk of $>15 \%$, and this threshold of coronary risk is reported in at least $60 \%$ of type II diabetics. ${ }^{9}$ In addition, there is limited evidence that statins may reduce retinal exudation in patients with diabetic maculopathy, an effect to be confirmed in ongoing large randomised clinical trials. ${ }^{15,16}$

This study has demonstrated that there is still much input required to achieve optimal metabolic and BP control to delay progression and need for laser treatment of DR. In view of the above findings, we feel that ophthalmologists responsible for the care of patients with DR should ensure that the patients receive both adequate medical therapies to optimise glycaemic and BP control, as well as laser photocoagulation. The importance of medical treatment is emphasised in patients with ischaemic maculopathy where laser treatment is largely ineffective. Although ophthalmologists may be concerned about involvement in medical therapeutic management, it is evident from our study that a simple electronic measurement of $\mathrm{BP}$ in the diabetic eye clinic is clinically useful, and should be influential in management.

\section{Conclusion}

This study has shown that BP recordings in diabetic eye clinics are of value, and should therefore influence clinical management. The results of casual diabetic eye clinic $\mathrm{BP}$ recordings were confirmed by home monitoring and $24 \mathrm{~h} \mathrm{ABPM}$. Despite the clear evidence of the importance of tight BP control in the prevention and progression of DR, our study has demonstrated that $72 \%$ of known hypertensive patients attending diabetic eye clinic are poorly controlled and $23 \%$ are new hypertensives.

Tight BP control in all patients' with DR is a goal yet to be achieved. This study emphasises that measurement of $\mathrm{BP}$ in the diabetic eye clinic should contribute to this goal.

\section{References}

1 Klein R, Klein BE, Moss SE, Davis MD, DeMets DL. The Wisconsin Epidemiologic Study of Diabetic Retinopathy. III. Prevalence and risk of diabetic retinopathy when age at diagnosis is 30 or more years. Arch Ophthalmol 1984; 102: 527-532.

2 Colhoun HM, Dong W, Barakat MT, Mather HM, Poulter NR. The scope for cardiovascular disease risk factor intervention among people with diabetes in England: a population-based analysis from the Health Surveys for England 1991-94. Diabet Med 1999; 16:35-40.

3 UK Prospective Diabetes Study Group. Tight blood pressure control and risk of macrovascular and microvascular complications in type 2 diabetes: UKPDS 38. BMJ 1998; 317: 703-713.

4 British Hypertension Society. Guidelines for management of hypertension: report of the third working party of the British Hypertension Society. J Hum Hypertens 1999; 13: 569-588.

5 O'Brien ET. Blood Pressure Measurement: Recommendations of the British Hypertension Society, 3rd ed. BMJ Publishing Group: London, 1997.

6 Stergiou GS, Skeya IL, Zourbaki AS, Mountokalakis TD. Self-monitoring of blood pressure at home: how many measurements are needed? J Hypertens 1998; 16: 725-731.

7 O'Brien E, Atkins N, Staessen J. State of the market. A review of ambulatory blood pressure monitoring devices. J Hypertens 1995; 26: 835-842.

8 The EDTRS Investigators. Aspirin effects on mortality and morbidity in patients with diabetes mellitus: Early Treatment Diabetic Retinopathy Study Report 14. JAMA 1992; 268: 1292-1300.

9 Bayly GR, Bartlett WA, Davies PH, Husband D, Haddon A, Game FL et al. Laboratory-based calculation of coronary heart disease risk in a hospital diabetic clinic. Diabet Med 1999; 16: 697-701.

10 Kohner EM, Patel V, Rassam SBM. Role of blood flow and impaired autoregulation in the pathogenesis of diabetic retinopathy. Diabetes 1995; 44: 603-607.

11 UK Prospective Diabetes Study (UKPDS) Group. Intensive blood glucose control with sulphonylureas or insulin compared with conventional treatment and risk of complications in patients with type 2 diabetes (UKPDS 33). Lancet 1998; 352: 837-885.

12 The Euclid Study Group. Randomised placebo-controlled trial of lisinopril in normotensive patients with insulindependent diabetes and normoalbuminuria or microalbuminuria. Lancet 1997; 349: 1787-1792.

13 Gillow JT, Gibson JM, Dodson PM. Hypertension and diabetic retinopathy - what's the story? Br J Ophthalmol 1999; 83: 1083-1087.

14 Hansson L, Zanchetti A, Carruthers SG, Dahlof B, Elmfeldt $\mathrm{D}$, Julius $\mathrm{S}$ et al. Effects of intensive blood pressure lowering and low-dose aspirin in patients with hypertension: principal results of the Hypertension Optimal Treatment (HOT) randomised trial. Hot Study Group. Lancet 1998; 352: 1755-1762.

15 Donaldson M, Dodson PM. The medical treatment of diabetic retinopathy. Eye (in press) 2003.

16 Chowdhury TA, Hopkins D, Dodson PM, Vafidis GC. The role of serum lipids in exudative diabetic maculopathy: is there a place for lipid lowering therapy? Eye 2002; 16: 689-693. 